\title{
Vortex states in iron-based superconductors with collinear antiferromagnetic cores
}

\author{
Hong-Min Jiang, ${ }^{1,2}$ Jian-Xin $\mathrm{Li}^{1}{ }^{1}$ and Z. D. Wang ${ }^{2}$ \\ ${ }^{1}$ National Laboratory of Solid State of Microstructure and Department of Physics, Nanjing University, Nanjing 210093, China \\ ${ }^{2}$ Department of Physics and Center of Theoretical and Computational Physics, The University of Hong Kong, \\ Pokfulam Road, Hong Kong, China \\ (Received 11 May 2009; revised manuscript received 14 August 2009; published 7 October 2009)
}

\begin{abstract}
Magnetism in the FeAs stoichiometric compounds and its interplay with superconductivity in vortex states are studied by self-consistently solving the Bogoliubov-de Gennes equations based on a two-orbital model with including the on-site interactions between electrons in the two orbitals. It is revealed that for the parent compound, magnetism is caused by the strong Hund's coupling, and the Fermi-surface topology aids to select the spin-density-wave (SDW) pattern. The superconducting (SC) order parameter with $s_{ \pm}=\Delta_{0} \cos \left(k_{x}\right) \cos \left(k_{y}\right)$ symmetry is found to be the most favorable pairing for both the electron- and hole-doped cases while the local density of states exhibits the characteristic of nodal gap for the former and full gap for the latter. In the vortex state, the emergence of the field-induced SDW depends on the strength of the Hund's coupling and the Coulomb repulsions. The field-induced SDW gaps the finite-energy contours on the electron- and hole-pocket sides, leading to the dual structures with one reflecting the SC pairing and the other being related to the SDW order. These features can be discernable in STM measurements for identifying the interplay between the field-induced SDW order and the SC order around the core region.
\end{abstract}

DOI: 10.1103/PhysRevB.80.134505 PACS number(s): 74.20.Mn, 74.25.Ha, 74.25.Jb, 74.72.Bk

\section{INTRODUCTION}

The recently discovered iron arsenide superconductors, ${ }^{1-5}$ which display superconducting transition temperature as high as more than $50 \mathrm{~K}$, appear to share a number of general features with high- $T_{c}$ cuprates, including the layered structure and proximity to a magnetically ordered state. ${ }^{1,6,7}$ The accumulated evidences have subsequently established a fact that the parent compounds are generally poor metal and undergo structure and antiferromagnetic (AFM) spin-densitywave (SDW) transitions below certain temperatures. ${ }^{6,8}$ Elastic neutron-scattering experiments have shown the antiferromagnetic order is collinear and has a wavevector $(\pi, 0)$ or $(0, \pi)$ in the unfolded Brillouin zone corresponding to a unit cell with only one $\mathrm{Fe}$ atom per unit cell. ${ }^{6}$ Either chemical doping or/and pressure suppresses the AFM SDW instability and eventually results in the emergence of superconductivity. ${ }^{1,9}$ The novel magnetism and superconducting properties in these compounds have been a great spur to recent researches. ${ }^{10-21}$

The relation between magnetism and superconductivity and the origin of magnetic order have attracted significant attentions in the current research on FeAs superconductors. Discrepancies exist in the experimental results, i.e., whether the superconductivity and antiferromagnetic order are well separated or they can coexist in the underdoped region of the phase diagram and how they coexist if they happen to do so. For example, there is no overlap between those two phases in $\mathrm{CeFeAsO}_{1-x} \mathrm{~F}_{x}$ (Ref. 10) while the coexistence of the two phases was observed in a narrow doping range in $\mathrm{SmFeAsO}_{1-x} \mathrm{~F}_{x}$ (Ref. 22) and in a broader range in $\mathrm{Ba}_{1-x} \mathrm{~K}_{x} \mathrm{Fe}_{2} \mathrm{As}_{2} \cdot{ }^{11,12}$ Even for the same $\mathrm{LaFeAsO}_{1-x} \mathrm{~F}_{x}$ system, different experiments display conflicting results. It was reported that before the orthorhombic SDW phase is completely suppressed by doping, superconductivity has already appeared at low temperatures, ${ }^{1}$ while it was also observed experimentally that superconductivity appears after the SDW is completely suppressed. ${ }^{13}$ As for the origin of the SDW phase, two distinct types of theories have been proposed: local-moment antiferromagnetic ground state for strong coupling $^{14}$ and itinerant ground state for weak coupling. ${ }^{15-18}$ The detection of the local moment seems to question the weak-coupling scenario but the metalliclike (or bad metal) nature as opposed to a correlated insulator as in cuprates renders the strong coupling theories doubtable. ${ }^{19}$ More recently, a compromised scheme was adopted: the SDW instability is assumed to result from the coupling of itinerant electrons with local moment, namely, neither the Fermi-surface nesting nor the local-moment scenario alone is able to account for it. ${ }^{20}$

Although many research efforts have been already made to identify the existence of magnetic order and its origin as well as the relationship with superconductivity, there have been fewer studies on vortex states of the systems. While the interplay between magnetism and superconductivity has been yet to be experimentally clarified, the superconducting critical temperature $T_{c}$ reaches its maximum value after the antiferromagnetic spin order is completely suppressed in the materials, indicating the competition nature between AFM SDW instability and superconductivity. At this stage, it is valuable and interesting to investigate vortex states in the family of FeAs compounds, mainly considering that the magnetic order may arise naturally when the superconducting order is destroyed by the magnetic vortex. Therefore, one can perform local tunneling spectroscopic probes in vortex states to understand profoundly the interplay between magnetic order and superconductivity.

In this paper, we investigate magnetism in the FeAs stoichiometric compounds, and the interplay between it and superconductivity upon doping in vortex states by selfconsistently solving the Bogoliubov-de Gennes (BdG) equations based on the two-orbital model with including the on-site interactions between electrons in the two orbitals. It 
is shown that for the parent compound, magnetism is caused by the strong Hund's coupling, and the Fermi-surface topology aids to select the SDW ordering pattern. The SDW results in the pseudogaplike feature at the Fermi level in the LDOS. It is found that the superconducting (SC) order parameter with $s_{ \pm}=\Delta_{0} \cos \left(k_{x}\right) \cos \left(k_{y}\right)$ symmetry is the most favorable pairing at both the electron- and hole-doped sides while the LDOS exhibits the characteristic of nodal gap for the former and full gap for the latter. In the vortex states, the emergence of the field-induced SDW order depends heavily on the strength of the Hund's coupling and the Coulomb repulsions. The coexistence of the field-induced SDW order and SC order around the core region is realized due to the fact that the two orders emerge at different energies. The corresponding LDOS at the core region displays a kind of dual structures with one reflecting the SC pairing and the other being related to the SDW order.

The paper is organized as follows. In Sec. II, we introduce the model Hamiltonian and carry out analytical calculations. In Sec. III, we present numerical calculations and discuss the results. In Sec. IV, we make remarks and conclusion.

\section{THEORY AND METHOD}

We start with an effective two-orbital model ${ }^{18}$ that takes only the iron $d_{x z}$ and $d_{y z}$ orbitals into account. By assuming an effective attraction that causes the superconducting pairing and including the possible interactions between the two orbitals' electrons, one can construct an effective model to study the vortex physics of the iron-based superconductors in the mixed state

$$
H=H_{0}+H_{\text {pair }}+H_{\text {int }} .
$$

The first term is a tight-binding model

$$
H_{0}=-\sum_{i j, \alpha \beta, \sigma} e^{i \varphi_{i j}} t_{i j, \alpha \beta} c_{i, \alpha, \sigma}^{\dagger} c_{j, \beta, \sigma}-\mu \sum_{i, \alpha, \sigma} c_{i, \alpha, \sigma}^{\dagger} c_{i, \alpha, \sigma},
$$

which describes the electron effective hoppings between sites $i$ and $j$ of the Fe ions on the square lattice, including the intraorbital $\left(t_{i j, \alpha \alpha}\right)$ and interorbital $\left(t_{i j, \alpha, \beta}, \alpha \neq \beta\right)$ hoppings with the subscripts $\alpha$ and $\beta[\alpha,(\beta)=1,2$ for $x z$ and $y z$ orbital, respectively] denoting the orbitals and $\sigma$ the spin. $c_{i, \alpha \sigma}^{\dagger}$ creates an $\alpha$ orbital electron with spin $\sigma$ at the site $i[i$ $\left.\equiv\left(i_{x}, i_{y}\right)\right]$, and $\mu$ is the chemical potential. The magnetic field is introduced through the Peierls phase factor $e^{i \varphi_{i j}}$ with $\varphi_{i j}$ $=\frac{\pi}{\Phi_{0}} \int_{r_{j}}^{r_{i}} \mathbf{A}(\mathbf{r}) \cdot d \mathbf{r}$, where $A=(-H y, 0,0)$ stands for the vector potential in the Landau gauge and $\Phi_{0}=h c / 2 e$ is the superconducting flux quantum. The hopping integrals are chosen as to capture the essence of the density function theory results. ${ }^{23}$ Taking the hopping integral between the $d_{y z}$ orbitals $\left|t_{1}\right|=1$ as the energy unit, we have

$$
\begin{gathered}
t_{i, i \pm \hat{x}, x z, x z}=t_{i, i \pm \hat{y}, y z, y z}=t_{1}=-1.0, \\
t_{i, i \pm \hat{y}, x z, x z}=t_{i, i \pm \hat{x}, y z, y z}=t_{2}=1.3, \\
t_{i, i \pm \hat{x} \pm \hat{y}, x z, x z}=t_{i, i \pm \hat{x} \pm \hat{y}, y z, y z}=t_{3}=-0.9, \\
t_{i, i+\hat{x}-\hat{y}, x z, y z}=t_{i, i+\hat{x}-\hat{y}, y z, x z}=t_{i, i-\hat{x}+\hat{y}, x z, y z}=t_{i, i+\hat{x}-\hat{y}, y z, x z}=t_{4}=-0.85,
\end{gathered}
$$

$$
t_{i, i+\hat{x}+\hat{y}, x z, y z}=t_{i, i+\hat{x}+\hat{y}, y z, x z}=t_{i, i-\hat{x}-\hat{y}, x z, y z}=t_{i, i-\hat{x}-\hat{y}, y z, x z}=-t_{4} .
$$

Here, $\hat{x}$ and $\hat{y}$ denote the unit vector along the $x$ and $y$ direction, respectively. The second term accounts for the superconducting pairing. Considering that a main purpose here is to address the interplay between the SC and magnetism in the vortex state for the FeAs-based superconductors, we take a phenomenological form for the pairing interaction

$$
H_{\text {pair }}=\sum_{i \neq j, \alpha \beta} V_{i j}\left(\Delta_{i j, \alpha \beta} c_{i, \alpha \uparrow}^{\dagger} c_{j, \beta \downarrow}^{\dagger}+\text { H.c. }\right)
$$

with $V_{i j}$ as the strengths of effective attractions.

The third term represents the interactions between electrons $^{24}$

$$
\begin{aligned}
H_{i n t}= & U \sum_{i, \alpha} n_{i, \alpha \uparrow} n_{i, \alpha \downarrow}+U^{\prime} \sum_{i, \alpha<\beta, \sigma} n_{i, \alpha, \sigma} n_{i, \beta, \bar{\sigma}} \\
& +\left(U^{\prime}-J\right) \sum_{i, \alpha<\beta, \sigma} n_{i, \alpha, \sigma} n_{i, \beta, \sigma} \\
& +J^{\prime} \sum_{i, \alpha<\beta}\left(c_{i, \alpha \uparrow}^{\dagger} c_{i, \alpha \downarrow}^{\dagger} c_{i, \beta \downarrow} c_{i, \beta \uparrow}+c_{i, \alpha \uparrow}^{\dagger} c_{i, \beta \downarrow}^{\dagger} c_{i, \alpha \downarrow} c_{i, \beta \uparrow}+\text { H.c. }\right),
\end{aligned}
$$

which includes the intraorbital (interorbital) Coulomb repulsion $U\left(U^{\prime}\right)$, the Hund's rule coupling $J$ as well as the interorbital Cooper pairing hopping term $J^{\prime}$.

After the Hartree-Fock decomposition of the on-site interaction term, one arrives at the Bogoliubov-de Gennes equations in the mean-field approximation for this model Hamiltonian

$$
\begin{aligned}
\sum_{j, \alpha<\beta}\left(\begin{array}{cccc}
H_{i j, \alpha \alpha, \sigma} & \tilde{\Delta}_{i j, \alpha \alpha} & H_{i j, \alpha \beta, \sigma} & \Delta_{i i, \beta \alpha}^{*} \\
\tilde{\Delta}_{i j, \alpha \alpha}^{*} & -H_{i j, \alpha \alpha, \bar{\sigma}}^{*} & \Delta_{i i, \alpha \beta} & -H_{i j, \alpha \beta, \bar{\sigma}}^{*} \\
H_{i j, \alpha \beta, \sigma} & \Delta_{i i, \alpha \beta}^{*} & H_{i j, \beta \beta, \sigma} & \tilde{\Delta}_{i j, \beta \beta} \\
\Delta_{i i, \beta \alpha} & -H_{i j, \alpha \beta, \bar{\sigma}}^{*} & \tilde{\Delta}_{i j, \beta \beta}^{*} & -H_{i j, \beta \beta, \bar{\sigma}}^{*}
\end{array}\right) \\
\times\left(\begin{array}{c}
u_{j, \alpha, \sigma}^{n} \\
v_{j, \alpha, \bar{\sigma}}^{n} \\
u_{j, \beta, \sigma}^{n} \\
v_{j, \beta, \bar{\sigma}}^{n}
\end{array}\right)=E_{n}\left(\begin{array}{l}
u_{i, \alpha, \sigma}^{n} \\
v_{i, \alpha, \bar{\sigma}}^{n} \\
u_{i, \beta, \sigma}^{n} \\
v_{i, \beta, \bar{\sigma}}^{n}
\end{array}\right),
\end{aligned}
$$

where

$$
\begin{gathered}
H_{i j, \alpha \alpha, \sigma}=-e^{i \varphi_{i j}} t_{i j, \alpha \alpha}+\delta_{i j}\left[U\left\langle n_{i, \alpha, \bar{\sigma}}\right\rangle+U^{\prime}\left\langle n_{i, \beta(\beta \neq \alpha), \bar{\sigma}}\right\rangle\right. \\
\left.+\left(U^{\prime}-J\right)\left\langle n_{i, \beta(\beta \neq \alpha), \sigma}\right\rangle-\mu\right], \\
H_{i j, \alpha \beta(\beta \neq \alpha), \sigma}=-e^{i \varphi_{i j}} t_{i j, \alpha \beta(\beta \neq \alpha)}, \\
\widetilde{\Delta}_{i j, \alpha \alpha}=\Delta_{i j, \alpha \alpha}+\Delta_{i i, \beta \beta(\beta \neq \alpha)}^{*} .
\end{gathered}
$$

$u_{j, \alpha, \sigma}^{n}\left(u_{j, \beta, \bar{\sigma}}^{n}\right)$ and $v_{j, \alpha, \sigma}^{n}\left(v_{j, \beta, \bar{\sigma}}^{n}\right)$ are the Bogoliubov quasiparticle amplitudes on the $j$ th site with corresponding eigenvalues $E_{n}$. 
The pairing amplitude and electron densities are obtained through the following self-consistent equations ${ }^{25}$

$$
\begin{gathered}
\Delta_{i j(i \neq j), \alpha \alpha}=\frac{V}{4} \sum_{n}\left(u_{i, \alpha, \sigma}^{n} v_{j, \alpha, \bar{\sigma}}^{n *}+v_{i, \alpha, \bar{\sigma}}^{n *} u_{j, \alpha, \sigma}^{n}\right) \times \tanh \left(\frac{E_{n}}{2 k_{B} T}\right), \\
\Delta_{i i, \alpha \beta}=\frac{J}{4} \sum_{n}\left(u_{i, \alpha, \sigma}^{n} v_{i, \beta, \bar{\sigma}}^{n *}+v_{i, \beta, \bar{\sigma}}^{n *} u_{i, \alpha, \sigma}^{n}\right) \times \tanh \left(\frac{E_{n}}{2 k_{B} T}\right), \\
n_{i, \alpha, \uparrow}=\sum_{n}\left|u_{i, \alpha, \uparrow}^{n}\right|^{2} f\left(E_{n}\right), \\
n_{i, \alpha, \downarrow}=\sum_{n}\left|v_{i, \alpha, \downarrow}^{n}\right|^{2}\left[1-f\left(E_{n}\right)\right] .
\end{gathered}
$$

The electronic structure associated with the SDW and the vortex states, namely, the local density of states (LDOS), $N\left(\mathbf{r}_{i}, E\right)$ is calculated by

$$
N\left(\mathbf{r}_{i}, E\right)=-\sum_{n, \alpha}\left[\left|u_{i, \alpha, \uparrow}^{n}\right|^{2} f^{\prime}\left(E_{n}-E\right)+\left|v_{i, \alpha, \downarrow}^{n}\right|^{2} f^{\prime}\left(E_{n}+E\right)\right],
$$

where $f^{\prime}(E)$ is the derivative of the Fermi-Dirac distribution function with respect to energy.

In numerical calculations, the undoped case is determined by the equality of the area enclosed by the electron and hole pocket in the unfolded Brillouin zone, which leads to $n_{h}$ $=\sum_{i, \alpha, \sigma}\left(n_{\alpha, \sigma}\right) /\left(N_{x} N_{y}\right)=2$. The Coulomb interactions $U, U^{\prime}, J, J^{\prime}$ are expected to satisfy the conventional relation $U^{\prime}=U-2 J$ and $J^{\prime}=J .{ }^{17,26}$ In the literatures, $U \sim(0.2$ $-0.5) W$ and $J \sim 0.09 W$ are expected. ${ }^{15,17}$ Here, $W$ is the energy bandwidth, which is $12.4\left|t_{1}\right|$ in our case. This gives rise to $J \sim\left|t_{1}\right|$ (Ref. 27) and $U \sim(2.2-5.5) J$. We have found numerically that the results presented here are not subject to the qualitative changes in the intermediate coupling range $U$ $\approx 3 \sim 4 J$, where the ground state is an AFM metal. ${ }^{28,29}$ In the following, the typical result with $U=3.5 \mathrm{~J}$ will be presented. We take $V_{i j}=0$ for the normal state. In the SC state, $V_{i j}$ is chosen to give a short coherence length of a few lattice spacing being consistent with experiments. ${ }^{30}$ We use $V_{i j}=V=2.0$ and $\mu=1.75(\mu=1.13)$, which gives rise to the filling factor $n=\sum_{i, \alpha, \sigma}\left(n_{\alpha, \sigma}\right) /\left(N_{x} N_{y}\right)=2.2(n=1.8)$ and the coherent peak of the SC order parameter in the density of state (DOS) being at $\Delta_{\text {max }} \simeq 0.4$. Thus, we estimate the coherence length $\xi_{0}$ $\sim E_{F} a /\left|\Delta_{\max }\right|$ (Ref. 31) $\sim 4 a$. Due to this short coherence length, presumably the system will be a type-II superconductor. The unit cell with size $N_{x} \times N_{y}=40 \times 20$ and the number of such unit cells $M_{x} \times M_{y}=10 \times 20$ are used in the numerical calculations. In view of these parameters, we estimate the upper critical field $B_{c 2} \sim 130 \mathrm{~T}$. Therefore, the model calculation is particularly suitable for the iron-based type-II superconductors such as $\mathrm{CaFe}_{1-x} \mathrm{Co}_{x} \mathrm{AsF}$, $\mathrm{Eu}_{0.7} \mathrm{Na}_{0.3} \mathrm{Fe}_{2} \mathrm{As}_{2}$, and $\mathrm{FeTe}_{1-x} \mathrm{~S}_{x}$, where the typical coherence length $\xi_{0}$ deduced from the experiments is of a few lattice spacing ${ }^{30}$ and the upper critical field achieves as high as dozens of Tesla. (a)

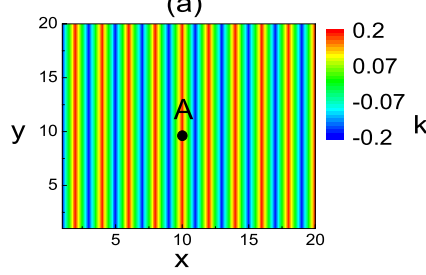

(c)

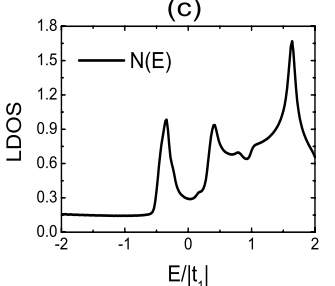

$E /\left|t_{1}\right|$ (b)

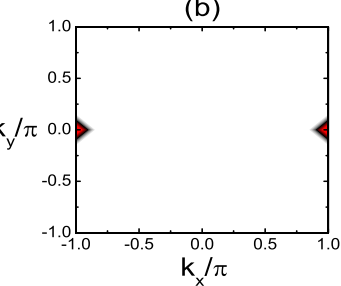

(d)

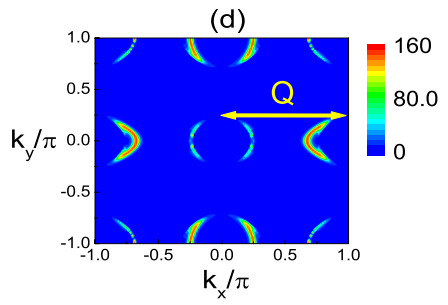

FIG. 1. (Color online) (a) The real-space distribution of the moment $M_{i}$. (b) The Fourier transformation of $M_{i}$. (c) The LDOS at the site labeled as A in Fig. 1(a) in the SDW state, and (d) spectralweight distribution in the SDW state (see text).

\section{RESULTS AND DISCUSSION}

\section{A. SDW phase in the absence of the magnetic field}

In the absence of a magnetic field and pairing term, we obtain the collinear AFM SDW at the half filling. Figure 1(a) is the typical result with $J=0.96$ for the real-space distribution of the moment $M_{i}$ defined as $M_{i}=\Sigma_{\alpha} M_{i, \alpha}$ with $M_{i, \alpha}$ $=\frac{1}{2}\left(\left\langle n_{i, \alpha, \uparrow}\right\rangle-\left\langle n_{i, \alpha, \downarrow}\right\rangle\right)$ being the spin order defined on the $\alpha$ orbital. As can be seen in Fig. 1(a), the real-space distribution of $M_{i}$ antiferromagnetically alines along the $x$ direction but ferromagnetically along the $y$ direction. In Fig. 1(b) Fourier transformation of $M_{i}$ gives an SDW order with wave vector $Q=(\pi, 0)$, which is consistent with experimental results in the undoped systems. ${ }^{6,32}$ [For another initial input parameters, the degenerate configuration of $M_{i}$ with wave vector $(0, \pi)$ can be obtained.] We note that the emergence of magnetic order is heavily dependent on the Hund's coupling strength $J$. For $J=0$, the magnetic-ordered phase does not exist even with very large $U$ and $U^{\prime}$. Therefore, magnetism itself is generated by the strong Hund's coupling, whereas the Fermi-surface topology aids to select the ordering pattern. ${ }^{33}$ This is a reminiscent of the spin freezing phase found in a three-orbital model relevant to transition-metal oxide $\mathrm{SrRuO}_{3}$ (Ref. 26) and may be a common feature respecting the magnetic-order origin in multiple orbital systems involving the Hund's coupling interaction.

In Fig. 1(c), we plot the LDOS $N\left(\mathbf{r}_{i}, E\right)$ in the SDW state at a site with positive $M_{i}$, i.e., the spin-up site labeled $A$ in Fig. 1(a). The electronic structure in the SDW state displays a clear pseudogaplike feature with a heavily depressed but nonvanishing DOS at the Fermi energy, pointing to the metallic magnetic ordered state. The magnetic order derived pseudogaplike feature is consistent with the experimental observation of partial gaps in the SDW state of the parent compounds $^{34}$ and may account for the pseudogap feature in several experiments. ${ }^{35,36}$

The pseudogap feature comes from a fact that when the SDW order with the wave vector $Q$ is involved, there will be 


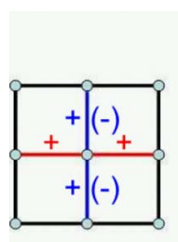

(a)

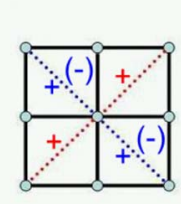

(b) (c)

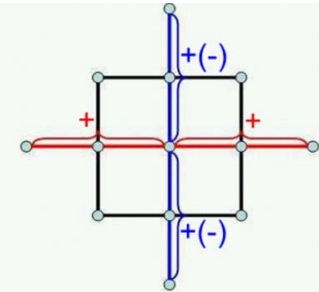

FIG. 2. (Color online) The SC order-parameter configuration in the (a) real space between the NN sites pairing, (b) the NNN sites pairing, (c) and the TNN sites pairing, respectively. The minus signs in the parentheses correspond to the $d$-wave pairings, otherwise the $s$-wave pairings.

gaps on those parts of the Fermi surface which are best connected by the wave vector $Q$, while those who are not connected by the wave vector $Q$ remain untouched, leading to the partial gaps in the SDW states of the parent compounds. ${ }^{37}$ We make this point more clear in Fig. 1(d), in which the spectral-weight distribution $I(k)$ $=\int_{\varepsilon_{F}-\Delta w}^{\varepsilon_{F}+\Delta w} A(k, \omega) d \omega$ is shown. Here, $A(k, \omega)$ is the singleparticle spectral function and $\Delta w$ is an integration window. As shown, both the electron- and hole-Fermi pockets are partially gaped.

\section{B. Configuration of the order parameters}

In the search for the most favorable pairing symmetry, we consider all possible singlet pairings, including the extended $s$ - and $d$-wave symmetries, between the nearest, next-nearest, and third-nearest-neighbor (NN, NNN, and TNN) sites, as shown in Figs. 2(a)-2(c). The pairing amplitude of the $s$-wave symmetry has the same sign along the $x$ and $y$ directions for the $\mathrm{NN}$ or TNN sites pairing, resulting in the $k$-dependent pairing form $\Delta(k)=\Delta_{0}\left[\cos \left(k_{x}\right)+\cos \left(k_{y}\right)\right]$ for the NN sites pairing and $\Delta(k)=\Delta_{0}\left[\cos \left(2 k_{x}\right)+\cos \left(2 k_{y}\right)\right]$ for the TNN sites pairing, respectively; and the same sign along the $x=y$ and $x=-y$ directions for the NNN sites pairing, resulting in the $k$-dependent pairing form $\Delta(k)=\Delta_{0}\left[\cos \left(k_{x}\right) \cos \left(k_{y}\right)\right]$. The $d$-wave pairing, on the other hand, has amplitude $+\Delta_{0}$ along the $x$ direction and $-\Delta_{0}$ along the $y$ direction for the NN or TNN sites pairing, resulting in the $k$-dependent pairing form $\Delta(k)=\Delta_{0}\left[\cos \left(k_{x}\right)-\cos \left(k_{y}\right)\right]$ for the NN sites pairing and $\Delta(k)=\Delta_{0}\left[\cos \left(2 k_{x}\right)-\cos \left(2 k_{y}\right)\right]$ for the TNN sites pairing, respectively; and $+\Delta_{0}$ along $x=y$ direction and $-\Delta_{0}$ along $x$ $=-y$ direction for the NNN sites pairing, resulting in the $k$-dependent pairing form $\Delta(k)=\Delta_{0}\left[\sin \left(k_{x}\right) \sin \left(k_{y}\right)\right]$.

The introduction of pairing interaction suppresses the SDW order completely on both the electron- and hole-doped sides, and leads to the homogeneous SC order in real space. We carry out extensive calculations and find that in the reasonable doping range the most favorable pairing symmetry is the intraorbital pairing between NNN sites

$$
\Delta_{i, i+\hat{x}+\hat{y}, \alpha \alpha}=\Delta_{i, i-\hat{x}-\hat{y}, \alpha \alpha}=\Delta_{i, i+\hat{x}-\hat{y}, \alpha \alpha}=\Delta_{i, i-\hat{x}+\hat{y}, \alpha \alpha}=\Delta_{i, \alpha \alpha},
$$

which leads to the $s_{ \pm}$-wave pairing $s_{ \pm}=\Delta_{0} \cos \left(k_{x}\right) \cos \left(k_{y}\right)$, being consistent with that obtained before. ${ }^{17,38,39}$ Then, the (a)

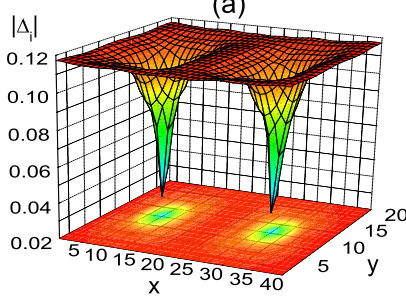

(c)

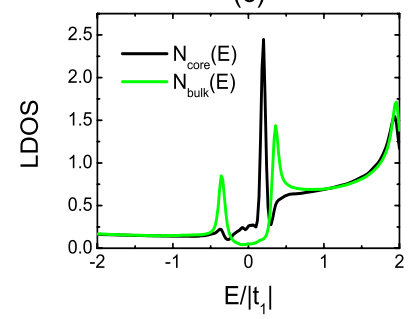

(b)

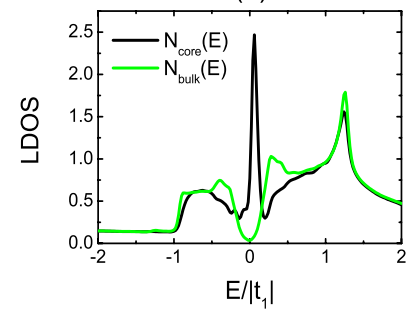

(d)

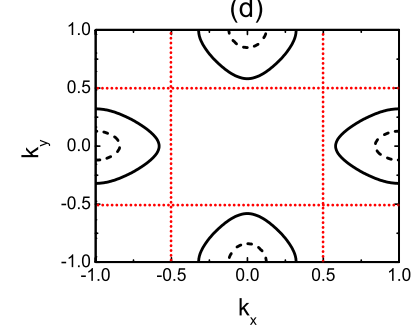

FIG. 3. (Color online) (a) The real-space distribution of the SC order amplitude $\left|\Delta_{i}\right|$ without the field-induced SDW. The LDOS curves at the core center (black line) and for the bulk system (green line) with (b) electron doping $n=2.2$ and (c) hole doping $n=1.8$, respectively. (d) The electron pocket in the iron pnictides in the unfolded Brillouin zone $-\pi \leq k_{x}<\pi$ and $-\pi \leq k_{y}<\pi$. The solid and dashed curves correspond to electron doping with $n=2.2$ and hole doping with $n=1.8$, respectively. The dotted (red) lines mark the nodal lines at $\left(\pi / 2, k_{y}\right)$ and $\left(k_{x}, \pi / 2\right)$ for the $s_{ \pm}$ $=\Delta_{0} \cos \left(k_{x}\right) \cos \left(k_{y}\right)$ order parameter.

superconducting order parameter $\Delta_{i}$ is expressed as

$$
\Delta_{i}=\frac{1}{2} \sum_{\alpha} \Delta_{i, \alpha \alpha} .
$$

For the choice of $V=2.0$ in this paper, one gets the amplitude $\Delta_{i} \sim 0.12$ for the $s_{ \pm} \mathrm{SC}$ order.

\section{Vortex states}

When a magnetic field is applied, the SC order parameter around the vortex core is suppressed so that the system may be driven into a vortex state. We find that there exists a critical Hund's coupling value $J_{c}$ separating the regimes of two kinds of vortex states associated, respectively, with and without the field-induced SDW order. In the following, we address these two regimes in detail.

\section{The vortex state without the field-induced SDW order}

The vortex state without the field-induced SDW order is stable when $J$ is less than $J_{c e}=0.9$ on the electron-doped side with $n=2.2$ and less than $J_{c h}=1.25$ on the hole-doped side with $n=1.8$, respectively. Typical results on the nature of the vortex state are displayed in Fig. 3 for $n=2.2$ with the Hund's coupling $J=0.85$, for which no magnetic order is induced. As shown in Fig. 3(a), each unit cell accommodates two superconducting vortices each carrying a flux quantum $h c / 2 e$. The SC order parameter $\Delta_{i}$ vanishes at the vortexcore center and recovers its bulk value at the core edge with radium $\xi_{1}$ on the scale of coherent length $\xi_{0}$. 
In Figs. 3(b) and 3(c) we plot the LDOS as a function of energy at the vortex-core center in the absence of the fieldinduced magnetic order for electron-doped case with $n=2.2$ and hole-doped case with $n=1.8$, respectively. For comparison, we have also displayed the LDOS at the midpoint between two nearest-neighbor vortices along the $x$ direction, which resembles that for the bulk system. As seen from Figs. 3(b) and 3(c), when $J=0.85$ for which no local SDW order is induced, the LDOS at the core center shows a single resonant peak within the SC gap edge for both the electron- and holedoped cases, which is similar to that reported by other authors for the cuprates high- $T_{c}$ superconductors in the vortex state. ${ }^{40}$ However, the differences are obvious with respect to the position of the resonant peak and the line shape of the bulk LDOS between the electron- and hole-doped cases in despite of the same SC pairing symmetry considered here. More specifically, for the electron-doped case, the position of the in-gap resonant peak is almost at the Fermi level and the bulk system exhibits the $V$-shaped LDOS curve, the typical characteristics which indicate a nodal SC gap. However, for the hole-doped case, the resonant peak deviates from the Fermi level to a higher energy and the bulk system exhibits the $U$-shaped LDOS curve, from which the conclusion for a full SC gap can be made.

The notable differences can be qualitatively understood as follows: the Fermi surface of the FeAs superconductors consists of hole Fermi surfaces around the $\Gamma$ point at $\left(k_{x}, k_{y}\right)$ $=(0,0)$ forming the hole pocket and the electron Fermi surfaces around the $M_{1,2}$ points at $(\pi, 0)$ and $(0, \pi)$ forming the electron pocket, respectively. Both Fermi pockets change their size upon doping as depicted in Fig. 3(d), where only the relevant electron pockets are displayed. The size of the electron pocket enlarges and approaches to the nodal line of the $s_{ \pm}$SC gap with electron doping while shrinks and deviates from the nodal line with hole doping. Thus the lowenergy quasiparticles in the SC phase show the nodal behavior in the electron-doped system and nodeless behavior in the hole-doped system. This may explain the discrepancy observed in experiments concerning the pairing symmetry, where the conclusion for the nodal gap were obtained on the electron-doped $L n \mathrm{FeAsO}$ ( $L n$ stands for the rare-earth elements) samples ${ }^{41-43}$ and a dominant full gap feature was found on the hole-doped $(\mathrm{Ba}, \mathrm{Sr})_{1-x} \mathrm{~K}_{x} \mathrm{Fe}_{2} \mathrm{As}_{2}$ systems $^{34,44}$ in the measurement of the thermal and transport properties.

\section{The vortex state with the field-induced SDW order}

As $J$ increases to about $J_{c e}=0.9$ on the electron-doped side with $n=2.2$ and $J_{c h}=1.25$ on the hole-doped side with $n=1.8$, the SDW order is induced around the core region. Figure 4 displays the vortex structure with $J=0.96$ for the electron-doped case, where the local magnetic order is induced around the vortex core, as shown in Fig. 4(b) which presents the spatial distribution of the local SDW order as defined in Sec. III A. As seen in Fig. 4(a), the vortex core expands further with a radium $\xi_{2}$ compared with that in Fig. 3(a). Meanwhile, the maximum strength of $M_{i}$ appears at the vortex-core center and decays with a scale of $\xi_{2}$ to zero into the superconducting region, depicting a competition nature
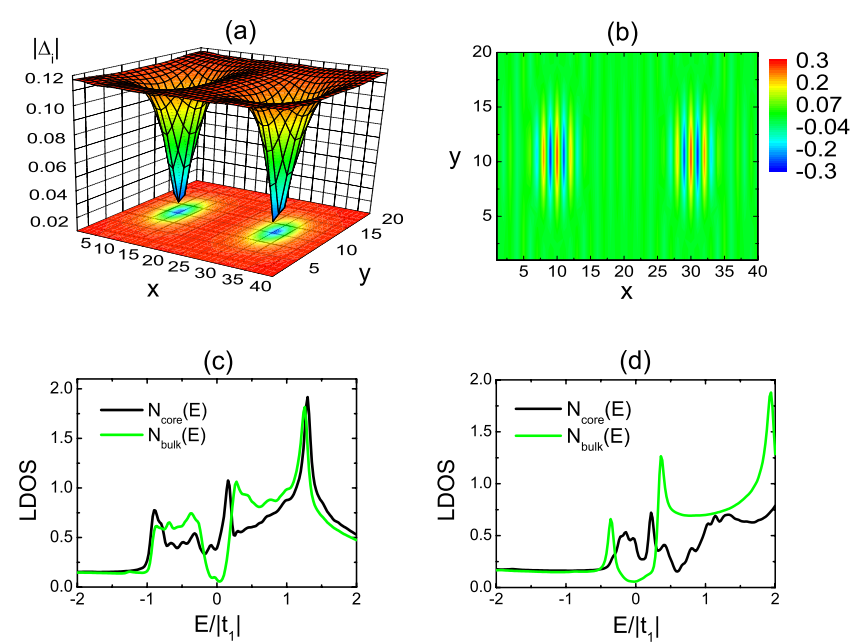

FIG. 4. (Color online) (a) The real-space distribution of the SC order amplitude $\left|\Delta_{i}\right|$ in the presence of the field-induced SDW. (b) The real-space distribution of the field-induced moment $M_{i}$. The LDOS curves at the core center (black line) and for the bulk system (green line) with (c) electron doping $n=2.2$ and (d) hole doping $n$ $=1.8$, respectively.

between SC and magnetic orders as observed in experiment. ${ }^{45}$

In this case, it is shown that there is no obvious splitting of the in-gap bound-state peak though the peak intensity is suppressed heavily in the LDOS for both the electron- and hole-doped cases, as shown in Figs. 4(c) and 4(d). In addition to the in-gap state peak, an additional peak structure below the Fermi energy appears for the electron-doped case, while two peaks situate separately around and above the Fermi energy for the hole-doped case. These features are dramatically different from the high- $T_{c}$ cuprates in vortex states with the field-induced antiferromagnetic order, where the in-gap resonant peak of the core bound state is split into two peaks sitting symmetrically about the Fermi energy. ${ }^{46}$

Simply, one can analyze the present vortex state in the following way: there are two factors that play a role in the physics around the vortex core region. One is the pure SC of vortex state without SDW in the magnetic field while the other is the SDW state without the SC order for the doped case. It is known that doping destroys the nesting property between parts of the Fermi surface on the electron and hole pocket due to the size change in the hole and electron pocket upon doping compared with that in the undoped case. However, as depicted in Figs. 5(c) and 5(d), the SDW wave vector $Q$ now connects the finite-energy contour for the doped case, resulting in the gaplike feature below (above) Fermi energy in the LDOS shown in Fig. 5(a) [Fig. 5(b)] for electron- (hole-)doped case. Combination of the in-gap resonant peak in the vortex state without SDW and the SDWinduced finite-energy gap feature produce a kind of dual structures of the LDOS for the finite doped case, i.e., the in-gap bound-state peak reflecting the SC pairing and the other peak structure being related to the SDW order.

\section{REMARKS AND CONCLUSION}

Clarification of the interplay between magnetism and superconductivity is a key step toward the understanding of the 
(a)

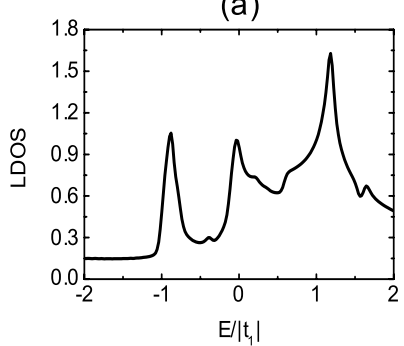

(c)

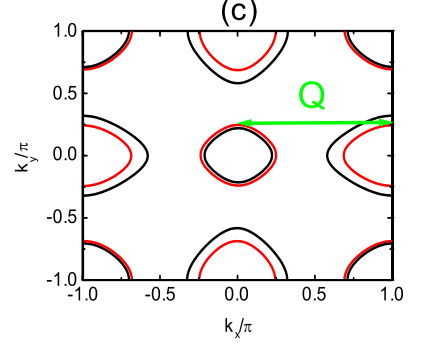

(b)

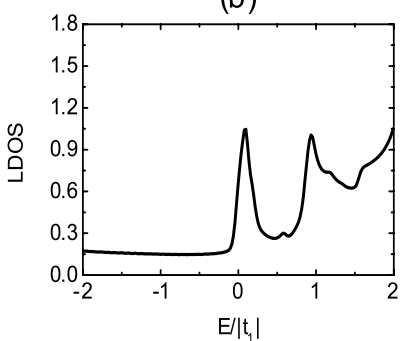

(d)

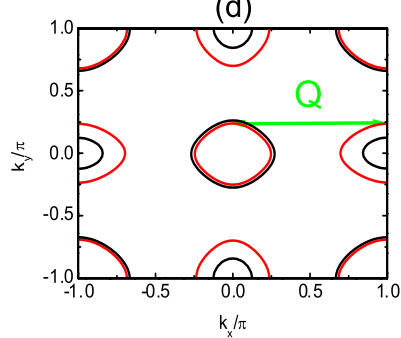

FIG. 5. (Color online) LDOS curves in a bulk system simulating the SDW state around the core region for $n=2.2$ (a), and $n=1.8$ (b), respectively. The wave vector $Q$ of the field-induced SDW connects the finite-energy contour (red line) for $n=2.2$ (c), and $n=1.8$, respectively (Black curves denote the Fermi surface at corresponding doping level).

underlying physics of the Fe-based high- $T_{c}$ superconductors. Although the competition nature between them has been identified in both classes of materials, some still show a coexistence of them..$^{11,12,22,45}$ Competition between the AFM SDW and SC is natural in FeAs compounds when one considers that both originate from the multiple Fe $d$ conduction bands but quest for the mechanism of the coexistence of them is shown to be more challenged. Recently, an incommensurate SDW state with wave vector $Q^{\prime}=Q \pm q$ has been proposed to account for the coexistence of the AFM SDW and superconductivity at finite doping. ${ }^{47,48}$ In such a state, the mismatch between the electron and hole Fermi pocket at finite doping is compensated by the incommensurate wave vector $q$, leading to the inferior "nesting" between the electron and hole Fermi pocket and allowing for the coexistence of magnetism and superconductivity. This mechanism only works at the doping level near the AFM instabilities, where the mismatch between the electron and hole Fermi pocket is small. Here, we show that the field-induced SDW at the doping level being far from the AFM instabilities is commensu-

rate with the same wave vector $Q$ as in the undoped case and it gaps the finite-energy contour on the electron- and holepocket sides. Therefore, at optimal doping, the field-induced SDW and the SC order around the core region may associate with the DOS at different energies, allowing them to coexist.

In conclusion, we have studied magnetism in the FeAs stoichiometric compounds and the interplay between it and superconductivity upon doping in the vortex state by selfconsistently solving the BdG equations based on the twoorbital model including the on-site interactions between electrons in the two orbitals. It has been shown that for the parent compound, magnetism is caused by the strong Hund's coupling, and the Fermi-surface topology aids to select the SDW ordering pattern. The SDW results in the pseudogaplike feature at the Fermi level in the LDOS. The SDW order is completely suppressed upon the introduction of the SC interaction. We have also found that the SC order parameter with $s_{ \pm}=\Delta_{0} \cos \left(k_{x}\right) \cos \left(k_{y}\right)$ symmetry is the most favorable pairing at both the electron- and hole-doped sides while the LDOS exhibits the characteristic of nodal gap for the former and full gap for the latter. In vortex states, the emergence of the field-induced SDW order depends heavily on the strength of the Hund's coupling and the Coulomb repulsions while the coexistence of the field-induced SDW order and SC order around the core region is realized due to the fact that the two orders emerge at different energies. The LDOS at the core region for the vortex state with SDW displays the dual structures with one reflecting the SC pairing and the other being related to the SDW order. These features can be discernable in the STM measurements for identifying the interplay between the field-induced SDW order and the SC order around the core region.

Note added. Recently, we have found a related work done by Hu et al. (Ref. 49).

\section{ACKNOWLEDGMENTS}

This project was supported by National Natural Science Foundation of China (Grants No. 10525415 and No. 10904062), the Ministry of Science and Technology of Science (Grants No. 2006CB601002 and 2006CB921800), a GRF grant of Hong Kong (Grant No. HKU7055/09P), the China Postdoctoral Science Foundation (Grant No. 20080441039), and the Jiangsu Planned Projects for Postdoctoral Research Funds (Grant No. 0801008C).
${ }^{1}$ Y. Kamihara, T. Watanabe, M. Hirano, and H. Hosono, J. Am. Chem. Soc. 130, 3296 (2008).

${ }^{2}$ X. H. Chen, T. Wu, G. Wu, R. H. Liu, H. Chen, and D. F. Fang, Nature (London) 453, 761 (2008).

${ }^{3}$ Z.-A. Ren, G.-C. Che, X.-L. Dong, J. Yang, W. Lu, W. Yi, X.-L. Shen, Z.-C. Li, L.-L. Sun, F. Zhou, and Z.-X. Zhao, EPL 83, 17002 (2008).

${ }^{4}$ G. F. Chen, Z. Li, D. Wu, G. Li, W. Z. Hu, J. Dong, P. Zheng, J. L. Luo, and N. L. Wang, Phys. Rev. Lett. 100, 247002 (2008).
${ }^{5}$ C. Wang, L. Li, S. Chi, Z. Zhu, Z. Ren, Y. Li, Y. Wang, X. Lin, Y. Luo, S. Jiang, X. Xu, G. Cao, and Z. Xu, EPL 83, 67006 (2008).

${ }^{6}$ C. de la Cruz, Q. Huang, J. W. Lynn, J. Li, W. Ratcliff II, J. L. Zarestky, H. A. Mook, G. F. Chen, J. L. Luo, N. L. Wang, and P. Dai, Nature (London) 453, 899 (2008).

${ }^{7}$ J. Dong, H. J. Zhang, G. Xu, Z. Li, G. Li, W. Z. Hu, D. Wu, G. F. Chen, X. Dai, J. L. Luo, Z. Fang, and N. L. Wang, EPL 83, 27006 (2008). 
${ }^{8}$ H.-H. Klauss, H. Luetkens, R. Klingeler, C. Hess, F. J. Litterst, M. Kraken, M. M. Korshunov, I. Eremin, S.-L. Drechsler, R. Khasanov, A. Amato, J. Hamann-Borrero, N. Leps, A. Kondrat, G. Behr, J. Werner, and B. Buchner, Phys. Rev. Lett. 101, 077005 (2008).

${ }^{9}$ H. Takahashi, K. Igawa, K. Arii, Y. Kamihara, M. Hirano, and H. Hosono, Nature (London) 453, 376 (2008).

${ }^{10}$ J. Zhao, Q. Huang, C. de la Cruz, S. Li, J. W. Lynn, Y. Chen, M. A. Green, G. F. Chen, G. Li, Z. Li, J. L. Luo, N. L. Wang, and P. Dai, Nature Mater. 7, 953 (2008).

${ }^{11}$ H. Chen, Y. Ren, Y. Qiu, Wei Bao, R. H. Liu, G. Wu, T. Wu, Y. L. Xie, X. F. Wang, Q. Huang, and X. H. Chen, EPL 85, 17006 (2009).

${ }^{12}$ T. Goko, A. A. Aczel, E. Baggio-Saitovitch, S. L. Bud'ko, P. C. Canfield, J. P. Carlo, G. F. Chen, P. Dai, A. C. Hamann, W. Z. Hu, H. Kageyama, G. M. Luke, J. L. Luo, B. Nachumi, N. Ni, D. Reznik, D. R. Sanchez-Candela, A. T. Savici, K. J. Sikes, N. L. Wang, C. R. Wiebe, T. J. Williams, T. Yamamoto, W. Yu, and Y. J. Uemura, Phys. Rev. B 80, 024508 (2009).

${ }^{13}$ H. Luetkens, H.-H. Klauss, M. Kraken, F. J. Litterst, T. Dellmann, R. Klingeler, C. Hess, R. Khasanov, A. Amato, C. Baines, M. Kosmala, O. J. Schumann, M. Braden, J. Hamann-Borrero, N. Leps, A. Kondrat, G. Behr, J. Werner, and B. Buechner, Nature Mater. 8, 305 (2009).

${ }^{14}$ Q. Si, and E. Abrahams, Phys. Rev. Lett. 101, 076401 (2008); Z. Y. Weng, Physica E (Amsterdam) 41, 1281 (2009); C. Xu, M. Müller, and S. Sachdev, Phys. Rev. B 78, 020501(R) (2008); C. Fang, H. Yao, W.-F. Tsai, J. P. Hu, and S. A. Kivelson, ibid. 77, 224509 (2008); G.-M. Zhang, Y.-H. Su, Z.-Y. Lu, Z.-Y. Weng, D.-H. Lee, and T. Xiang, EPL 86, 37006 (2009).

${ }^{15}$ C. Cao, P. J. Hirschfeld, and H.-P. Cheng, Phys. Rev. B 77, 220506(R) (2008); S. Yang, W.-L. You, S.-J. Gu, and H.-Q. Lin, Chin. Phys. B 18, 2545 (2009).

${ }^{16}$ D. J. Singh and M. H. Du, Phys. Rev. Lett. 100, 237003 (2008); K. Kuroki, S. Onari, R. Arita, H. Usui, Y. Tanaka, H. Kontani, and H. Aoki, ibid. 101, 087004 (2008); Q. Han, Y. Chen, and Z. D. Wang, EPL 82, 37007 (2008); Q. Han and Z. D. Wang, New J. Phys. 11, 025022 (2009); V. Cvetkovic and Z. Tesanovic, EPL 85, 37002 (2009).

${ }^{17}$ Z.-J. Yao, J.-X. Li, and Z. D. Wang, New J. Phys. 11, 025009 (2009); S. L. Yu, J. Kang, and J. X. Li, Phys. Rev. B 79, 064517 (2009).

${ }^{18}$ S. Raghu, X.-L. Qi, C.-X. Liu, D. J. Scalapino, and S.-C. Zhang, Phys. Rev. B 77, 220503(R) (2008).

${ }^{19}$ W. Z. Hu, J. Dong, G. Li, Z. Li, P. Zheng, G. F. Chen, J. L. Luo, and N. L. Wang, Phys. Rev. Lett. 101, 257005 (2008).

${ }^{20}$ J. Dai, Q. Si, J.-X. Zhu, and E. Abrahams, Proc. Natl. Acad. Sci. U.S.A. 106, 4118 (2009); S.-P. Kou, T. Li, and Z.-Y. Weng, arXiv:0811.4111 (unpublished).

${ }^{21}$ Y. Wan and Q.-H. Wang, EPL 85, 57007 (2009).

${ }^{22}$ R. H. Liu, G. Wu, T. Wu, D. F. Fang, H. Chen, S. Y. Li, K. Liu, Y. L. Xie, X. F. Wang, R. L. Yang, L. Ding, C. He, D. L. Feng, and X. H. Chen, Phys. Rev. Lett. 101, 087001 (2008); A. J. Drew, F. L. Pratt, T. Lancaster, S. J. Blundell, P. J. Baker, R. H. Liu, G. Wu, X. H. Chen, I. Watanabe, V. K. Malik, A. Dubroka, K. W. Kim, M. Rössle, and C. Bernhard, ibid. 101, 097010 (2008).

${ }^{23}$ G. Xu, W. Ming, Y. Yao, X. Dai, S.-C. Zhang, and Z. Fang, EPL 82, 67002 (2008); Z. P. Yin, S. Lebègue, M. J. Han, B. P. Neal, S. Y. Savrasov, and W. E. Pickett, Phys. Rev. Lett. 101, 047001
(2008).

${ }^{24}$ A. M. Oleś, G. Khaliullin, P. Horsch, and L. F. Feiner, Phys. Rev. B 72, 214431 (2005).

${ }^{25}$ Y. Chen, Z. D. Wang, J.-X. Zhu, and C. S. Ting, Phys. Rev. Lett. 89, 217001 (2002).

${ }^{26}$ P. Werner, E. Gull, M. Troyer, and A. J. Millis, Phys. Rev. Lett. 101, 166405 (2008).

${ }^{27}$ K. Haule and G. Kotliar, New J. Phys. 11, 025021 (2009).

${ }^{28}$ J. Lorenzana, G. Seibold, C. Ortix, and M. Grilli, Phys. Rev. Lett. 101, 186402 (2008).

${ }^{29}$ R. Yu, K. T. Trinh, A. Moreo, M. Daghofer, J. A. Riera, S. Haas, and E. Dagotto, Phys. Rev. B 79, 104510 (2009).

${ }^{30}$ S. Takeshita, R. Kadono, M. Hiraishi, M. Miyazaki, A. Koda, S. Matsuishi, and H. Hosono, Phys. Rev. Lett. 103, 027002 (2009); Y. Qi, Z. Gao, L. Wang, D. Wang, X. Zhang, and Y. Ma, New J. Phys. 10, 123003 (2008); Y. Mizuguchi, F. Tomioka, S. Tsuda, T. Yamaguchi, and Y. Takano, Appl. Phys. Lett. 94, 012503 (2009).

${ }^{31}$ Y. D. Zhu, F. C. Zhang, and M. Sigrist, Phys. Rev. B 51, 1105 (1995).

${ }^{32}$ Y. Chen, J. W. Lynn, J. Li, G. Li, G. F. Chen, J. L. Luo, N. L. Wang, P. Dai, C. de la Cruz, and H. A. Mook, Phys. Rev. B 78, 064515 (2008)

${ }^{33}$ I. I. Mazin and J. Schmalian, Physica C 469, 614 (2009).

${ }^{34}$ W. Z. Hu, Q. M. Zhang, and N. L. Wang, Physica C 469, 545 (2009).

${ }^{35}$ H.-Y. Liu, X.-W. Jia, W.-T. Zhang, L. Zhao, J.-Q. Meng, G.-D. Liu, X.-L. Dong, G. Wu, R.-H. Liu, X.-H. Chen, Z.-A. Ren, W. Yi, G.-C. Che, G.-F. Chen, N.-L. Wang, G.-L. Wang, Y. Zhou, Y. Zhu, X.-Y. Wang, Z.-X. Zhao, Z.-Y. Xu, C.-T. Chen, and X.-J. Zhou, Chin. Phys. Lett. 25, 3761 (2008); L. Zhao, H.-Y. Liu, W.-T. Zhang, J.-Q. Meng, X.-W. Jia, G.-D. Liu, X.-L. Dong, G.-F. Chen, J.-L. Luo, N.-L. Wang, W. Lu, G.-L. Wang, Y. Zhou, Y. Zhu, X.-Y. Wang, Z.-Y. Xu, C.-T. Chen, and X.-J. Zhou, ibid. 25, 4402 (2008).

${ }^{36}$ M. C. Boyer, K. Chatterjee, W. D. Wise, G. F. Chen, J. L. Luo, N. L. Wang, and E. W. Hudson, arXiv:0806.4400 (unpublished); T. Sato, S. Souma, K. Nakayama, K. Terashima, K. Sugawara, T. Takahashi, Y. Kamihara, M. Hirano, and H. Hosono, J. Phys. Soc. Jpn. 77, 063708 (2008); Y. Ishida, T. Shimojima, K. Ishizaka, T. Kiss, M. Okawa, T. Togashi, S. Watanabe, X. Y. Wang, C. T. Chen, Y. Kamihara, M. Hirano, H. Hosono, and S. Shin, Phys. Rev. B 79, 060503(R) (2009).

${ }^{37}$ D. Hsieh, Y. Xia, L. Wray, D. Qian, K. K. Gomes, A. Yazdani, G. F. Chen, J. L. Luo, N. L. Wang, and M. Z. Hasan, arXiv:0812.2289 (unpublished).

${ }^{38}$ I. I. Mazin, D. J. Singh, M. D. Johannes, and M. H. Du, Phys. Rev. Lett. 101, 057003 (2008); K. Seo, B. A. Bernevig, and J. P. Hu, ibid. 101, 206404 (2008).

${ }^{39}$ F. Wang, H. Zhai, Y. Ran, A. Vishwanath, and D. H. Lee, Phys. Rev. Lett. 102, 047005 (2009); W.-Q. Chen, K.-Y. Yang, Y. Zhou, and F.-C. Zhang, ibid. 102, 047006 (2009); A. V. Chubukov, Physica C 469, 640 (2009).

${ }^{40}$ Y. Wang and A. H. MacDonald, Phys. Rev. B 52, R3876 (1995); M. Franz and Z. Tešanović, Phys. Rev. Lett. 80, 4763 (1998).

${ }^{41}$ G. Mu, X. Y. Zhu, L. Fang, L. Shan, C. Ren, and H. H. Wen, Chin. Phys. Lett. 25, 2221 (2008); L. Shan, Y. L. Wang, X. Y. Zhu, G. Mu, L. Fang, C. Ren, and H. H. Wen, EPL 83, 57004 (2008); C. Ren, Z. S. Wang, H. Yang, X. Y. Zhu, L. Fang, G. $\mathrm{Mu}, \mathrm{L}$. Shan, and H. H. Wen, arXiv:0804.1726 (unpublished). 
${ }^{42}$ H.-J. Grafe, D. Paar, G. Lang, N. J. Curro, G. Behr, J. Werner, J. Hamann-Borrero, C. Hess, N. Leps, R. Klingeler, and B. Buchner, Phys. Rev. Lett. 101, 047003 (2008); H. Luetkens, H.-H. Klauss, R. Khasanov, A. Amato, R. Klingeler, I. Hellmann, N. Leps, A. Kondrat, C. Hess, A. Köhler, G. Behr, J. Werner, and B. Büchner, ibid. 101, 097009 (2008); J. P. Carlo, Y. J. Uemura, T. Goko, G. J. MacDougall, J. A. Rodriguez, W. Yu, G. M. Luke, P. C. Dai, N. Shannon, S. Miyasaka, S. Suzuki, S. Tajima, G. F. Chen, W. Z. Hu, J. L. Luo, and N. L. Wang, ibid. 102, 087001 (2009).

${ }^{43}$ K. Matano, Z. A. Ren, X. L. Dong, L. L. Sun, Z. X. Zhao, and G. Q. Zheng, EPL 83, 57001 (2008); K. Ahilan, F. L. Ning, T. Imai, A. S. Sefat, R. Jin, M. A. McGuire, B. C. Sales, and D. Mandrus, Phys. Rev. B 78, 100501(R) (2008); F. Massee, Y. Huang, R. Huisman, S. de Jong, M. S. Golden, and J. B. Goedkoop, ibid. 79, 220517(R) (2009).
${ }^{44}$ G. Li, W. Z. Hu, J. Dong, Z. Li, P. Zheng, G. F. Chen, J. L. Luo, and N. L. Wang, Phys. Rev. Lett. 101, 107004 (2008); G. Mu, H. Luo, Z. Wang, L. Shan, C. Ren, and Hai-Hu Wen, Phys. Rev. B 79, 174501 (2009).

${ }^{45}$ D. K. Pratt, W. Tian, A. Kreyssig, J. L. Zarestky, S. Nandi, N. Ni, S. L. Bud'ko, P. C. Canfield, A. I. Goldman, and R. J. McQueeney, Phys. Rev. Lett. 103, 087001 (2009).

${ }^{46}$ I. Maggio-Aprile, Ch. Renner, A. Erb, E. Walker, and Ø. Fischer, Phys. Rev. Lett. 75, 2754 (1995); J.-X. Zhu and C. S. Ting, ibid. 87, 147002 (2001).

${ }^{47}$ V. Cvetkovic and Z. Tesanovic, Phys. Rev. B 80, 024512 (2009).

${ }^{48}$ A. B. Vorontsov, M. G. Vavilov, and A. V. Chubukov, Phys. Rev. B 79, 060508(R) (2009).

${ }^{49}$ X. Hu, C. S. Ting, and J. X. Zhu, Phys. Rev. B 80, 014523 (2009). 